\title{
LARVES D'GESTRIDES
}

\section{PRODUISANT DES TUMEURS DUODÉNALES \\ CHEZ LES CHEVAUX EN ESPAGNE}

\author{
Par G. DINULESCU
}

Tous les gastrophiles parasites du cheval décrits jusqu'à présent vivent, pendant leurs $2^{\circ}$ et $3^{\circ}$ stades larvaires, fixés à la surface du tube digestif de l'hôte, chaque espèce se localisant en une région déterminée. Les larves, avant d'arriver dans l'estomac, l'intestin ou le rectum, restent pendant toute la durée du $1^{\text {er }}$ stade enfoncées dans la muqueuse buccale (Roubaud, 1917, p. 453 ; Hobmeyer, 1928, p. 163 ; Dinulescu, 1930 , p. 491 ) et on les trouve dans le pharynx au début du $2^{\circ}$ stade. De là, les larves vont se fixer : celles du Gastrophilus intestinalis (de G.) toujours dans l'estomac; celles du G. hæmorrhoidalis L. dans l'estomac et le rectum ; celles du G. pecorum Fbr. dans l'estomac et quelquefois dans le duodenum. Les larves du G. nasalis Clark se fixent dans le duodenum et celles du G. inermis $\mathrm{Br}$. dans le rectum. Les maladies produites par les gastrophiles sont les suivantes : myiase gastrophilienne des joues du cheval, causée par les larves primaires du Gastrophilus inermis (Dinulescu, 1929, p. 419) ; myiases de l'estomac, de l'intestin grêle et du rectum produites par les larves au $2^{\circ}$ et au $3^{\circ}$ stades de tous les gastrophiles. Les larves, en se fixant à la surface interne de l'estomac, de l'intestin et du rectum, produisent des lésions alvéolaires, des ulcérations et quelquefois des déchirures de la paroi. Ces larves, surtout au $1^{\mathrm{e}_{\mathrm{r}}}$ stade, en passant par la bouche et le pharynx de l'hôte, produisent des lésions sinueuses et fistuleuses dans la muqueuse de ces organes. Outre ces lésions classiques, on a signalé deux cas particuliers de myiases provoquées par des larves d'autres œestrides. Un cas (Brauer, 1864, p. 891) où une larve d'œstride (CEstromyia spp ?) a été trouvée dans une tumeur de l'abdomen d'un campagnol, Hypudeus arvalis ; un autre cas (Girard, 1874, p. 3) où une larve d'hypoderme (Hypoderma sp ?) était logée dans une grande tumeur chez une sarigue (Didelphis murina). Enfin, comme action pathogène spéciale des larves de gastrophiles, citons les conclusions de Goyanez (1926, p. 197) qui, dans une étude sur l'influence des parasites

Annales de Parasitologie, t. IX, $x^{\circ} 6 .-1^{\text {er }}$ novembre 1931 , p. 503-511. 
animaux dans la genèse du cancer, signale que les larves de gastrophiles peuvent pénétrer dans la muqueuse gastrique des chevaux et y produire des lésions qui peuvent dégénérer en carcinome.

Dans ce mémoire, nous signalons une larve d'œstride qui est fixée au $3^{\text {e }}$ stade dans le duodenum du cheval ; auparavant, elle passe une partie de sa vie ( $2^{e}$ stade larvaire) dans la profondeur des tissus de la paroi de l'intestin, en provoquant la formation de certaines tumeurs spécifiques. Nous avons trouvé cètte larve parasitant très fréquemment les chevaux provenant de l'Espagne. Jamais nous ne l'avons trouvée sur les chevaux provenant de France ou de l'Europe centrale, de sorte que l'espèce à laquelle elle appartient doit avoir une aire géographique méridionale.

Cet été, les abattoirs de Vaugirard ont reçu beaucoup de chevaux venant d'Espagne, ce qui nous a permis d'avoir un matériel suffisant pour suivre une partie de la vie de cette larve et en même temps pour étudier ces formations tumorales dans la paroi du duodenum. Cette larve, que nous avons pu suivre pendant deux stades larvaires, parait se rapprocher beaucoup (surtout au $3^{\circ}$ stade) d'une autre forme d'œstride signalée dans l'estomac des zèbres de Rhodesia (Afrique du Sud) et qui a été décrite par Pillers et Ewans sous le nom d'CEstrus (Gastrophilus) meridionalis.

Nous avons été amené à découvrir cette larve en cherchant chez les chevaux des larves de Gastrophilus nasalis Clark. Nous avons remarqué, à un moment donné, que certains chevaux présentaient, dans le duodénum, non seulement des larves, mais encore, dans la paroi de cet organe, quelques renflements durs de volume variable. A l'ouverture, ces formations d'aspect fibreux, laissaient voir de nombreuses larves jeunes. Nous avons remarqué ensuite que dans le groupe de larves fixées à la surface et que nous supposions appartenir au Gastrophilus nasalis, il y avait une forme larvaire très différente.

\section{Description DE LA LARVE DU Gastrophilus meridionalis}

Larve au $2^{\text {e }}$ stade (fig. 1). - Nous décrirons ici la larve au stade où on la trouve à l'intérieur des tumeurs de la paroi duodénale. La larve est composée de 13 segments. Tous les segments sont visibles, le $13^{\circ}$ n'étant pas replié à l'intérieur du $12^{\circ}$. Les dimensions sont variables suivant l'âge de la larve. Considérée au moment où elle prépare sa mue pour le $3^{\circ}$ stade, la larve a une longueur de 14 à $16 \mathrm{~mm}$. et une largeur de 3 à $4 \mathrm{~mm}$. dans la partie 
moyenne du corps. Elle a une forme cylindrique et parait plus allongée que les larves des autres espèces. Sa couleur est blan-

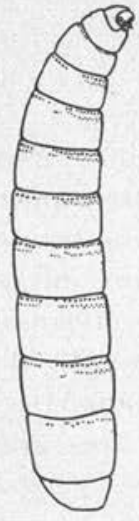

FIG. 1. - Larve de G. meridionalis au $2^{\circ}$ stade, vue latérale$\mathrm{ment}$. Même échelle que pour la fig. 2. chàtre.

Tous les segments, du $2^{\circ}$ au $12^{\circ}$, présentent des épines disposées en rangées contournant chaque segment. Ces épines, à la différence de ce qu'on observe chez toutes les autres espèces, sont très réduites et presque atrophiées ; elles sont disposées en 3 rangées sur la partie ventrale et en 2 rangées sur la partie dorsale de chaque segment. Ces épines manquent sur la partie dorsale du $12^{\circ}$ segment, et elles sont presque invisibles sur les $3^{\circ}$ et $4^{\circ}$ segments (fig. 2, pl. X). Les pièces buccales, composées de deux crochets mandibulaires et de deux mâchoires, sont bien développées. Les stigmates qui se trouvent sur le $13^{\mathrm{e}}$ segment sont très réduits et presque atrophiés. Macroscopiquement, ils sont réduits à deux points. Mais, à un faible grossissement, ils montrent deux paires de fentes courtes non recourbées et dont l'ouverture présente un bord très irrégulier (fig. 6).

Larve au $3^{\mathrm{e}}$ stade (fig. 2). - A ce stade la larve présente toujours 13 segments, mais le premier segment céphalique, très court, fait corps avec le $2^{\circ}$ segment. Le $13^{\circ}$ segment, sur lequel s'ouvrent les stigmates postérieurs, est replié à l'intérieur du $12^{\circ}$; chez la larve au repos, morte ou fixée, le corps parait être divisé en 11 segments. Dimensions : longueur, 16 à $18 \mathrm{~mm}$. ; largeur dans la partie moyenne du corps, 6-7 $\mathrm{mm}$. La couleur est généralement jaune pâle. La cuticule de la larve est presque transparente sur la partie dorsale du corps de sorte qu'on aperçoit la couleur rouge du sang ingéré.

Les épines chitineuses sont disposées sur les divers segments en une seule rangée, les $3^{\text {e }}$ et $4^{\circ}$ segments étant dépourvus de tout ornement chitineux. Le $3^{\circ}$ segment présente encore trois ou quatre plis longitudinaux sur les parties dorsale et ventrale et, entre le $3^{\circ}$ et le $4^{\circ}$ segments, il y a un étranglement prononcé. Les stigmates, normalement développés à ce

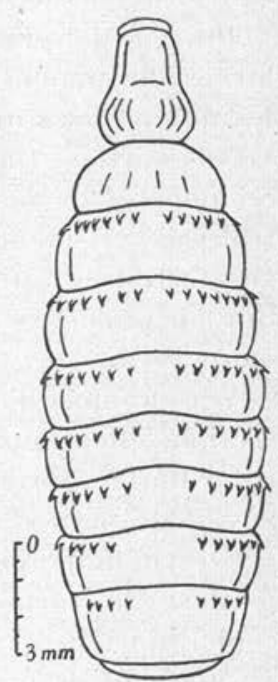

Fig. 2. - Larre de Gastrophilus meridionalis (?) au 3 ' stade, face dorsale. 
stade, sont disposés sur une plaque portant trois paires de fentes légèrement recourbées et laissant au milieu de la plaque un espace de forme losangique (fig. 5). Les pièces buccales sont semblables à celles du second stade.

\section{Evolution DE LA LARVE ET PRODUCTION DES TUMEURS}

Nos observations ont commencé au mois de mai de cette année et elles ont duré jusqu'à la fin du mois d'août, date à laquelle les arrivages de chevaux d'Espagne à Vaugirard étaient de plus en plus rares. Grâce à l'aimable autorisation de M. Raymond, vétérinairedirecteur des abattoirs, et avec le précieux concours de M. Blanchard, vétérinaire-inspecteur, nous avons pu prélever la partie du duodénum contenant des larves et des tumeurs sur un très grand nombre de chevaux provenant d'Espagne.

Sur ces nombreuses pièces, les larves et les tumeurs ont préser ‘é des différences suivant la période à laquelle les observations ont été faites.

Au début du mois de mai, presque toutes ces tumeurs contenaient de nombreuses larves au $2^{\text {e }}$ stade et d'àge différent. Une grande partie de ces larves présentaient au-dessous de leur tégument la cuticule du stade suivant (fig. 3 , pl. XI). A la surface interne de l'intestin, il y avait toujours un groupe de larves au $3^{\text {e }}$ stade.

Plus tard, vers le mois de juin et mêmé au début de juillet, les larves devenaient plus rares à l'intérieur des tumeurs. Mais, dans les nombreuses loges vides, il y avait des mues abandonnées par les larves sorties. Dans le groupe de larves de la surface, il y avait à ce moment de nombreuses larves au $3^{e}$ stade bien développées. Je trouvais en même temps, à l'intérieur des tumeurs, de jeunes larves tertiaires qui cherchaient à sortir. Les tumeurs présentaient une ou plusieurs ouvertures et de nombreux canaux pleins de pus. Dans ce pus il y avait également des mues larvaires.

Vers le mois d'aqût les tumeurs étaient de plus en plus vides. A la section, elles montraient des loges vides, mais obstruées par le tissu fibreux environnant. Dans la masse fibreuse de la tumeur, on voyait de petites taches rondes, de couleur rougeâtre, dues à de petites hémorragies, produites par les larves. D’autres taches, plus petites, de forme irrégulière, marquaient l'armature chitineuse céphalique du $2^{\circ}$ stade dans la dépouille abandonnée par les larves au moment de la mue. A la surface interne, il $\mathrm{y}$ avait à ce moment de rares larves au $3^{\text {e }}$ stade, de plus en plus développées et, à côté de celles-ci, on voyait un nombre considérable de très jeunes larves 
secondaires qui devaient pénétrer plus tard dans les tissus de la paroi de l'organe (fig. 8 , pl. X).

D'après ces observations, nous pouvons résumer l'évolution larvaire de cet ostride de la façon suivante : les larves, après avoir passé leur $1^{\text {er }}$ stade dans les muqueuses buccale et pharyngienne, viennent se fixer, sous la forme du $2^{\circ}$ stade, dans l'intestin sur la muqueuse duodénale. Là, elles cherchent à s'enfoncer dans la profondeur de la paroi ; probablement par irritation, elles déterminent une réaction locale des tissus qui se traduit par la formation d'un tissu fibreux qui les entoure comme une sorte de loge, dans laquelle on peut trouver de 2 à 5 larves. Dans les grosses tumeurs, il peut mème y en avoir plus d'une vingtaine, dispersées dans la masse fibreuse.

Les larves grandissent à l'intérieur des tumeurs et, arrivées à un certain âge, elles muent sur place et abandonnent la dépouille du $2^{\circ}$ stade. Arrivées au $3^{\circ}$ stade, elles sortent dans la cavité intestinale où elles se fixent de nouveau à la paroi en y implantant profondément la partie antérieure de leur corps. Constamment, les larves de cette espèce enfoncent dans la paroi de l'intestin la tête et les deux segments suivants qui sont dépourvus d'épines. Elles produisent alors des lésions alvéolaires très profondes.

On voit donc que, pendant leur vie, ces larves présentent cette curieuse adaptation à une vie anaérobie à l'intérieur de tumeurs où elles sont séparées de l'air atmosphérique d'où elles pourraient puiser l'oxygène nécessaire à leur respiration. Cette question d'anaérobiose a déjà été soulevée par Kemnitz (1917) pour les larves de gastrophiles qui vivent fixées à la surface du tube digestif. D'ailleurs, nous reviendrons sur cette question dans une prochaine publication. Morphologiquement, cette adaptation se traduit par la réduction sensible des orifices respiratoires (stigmates postérieurs), par une mobilité très restreinte du corps de la larve et par l'atrophie des épines chitineuses qui ornent la surface du corps de ces larves.

Caractères des tumeurs. - Les tumeurs sont de dimensions variant de la grosseur d'une noix ou même au-dessous, jusqu'à celle d'un gros œuf de poule ; elles peuvent être au nombre d'une ou plusieurs chez un même animal. Elles se forment toujours dans la paroi duodénale près du pylore, quelquefois mème elles peuvent se trouver dans l'épaisseur du sphincter pylorique. Les petites tumeurs ont toujours une forme arrondie et font saillie à la surface interne du duodénum. Les grosses tumeurs font saillie à la face externe 
de l'intestin. Ces tumeurs sont percées d'un ou de plusieurs orifices qui traversent la muqueuse intestinale. Sur la coupe, on voit, à l'intérieur de la masse fibreuse qui remplit ces tumeurs, de petites loges ou des canaux où se trouvent les larves au $2^{\circ}$ stade. Les tumeurs sont d'autant plus grandes qu'il y a plus de larves. Les petites renferment 2 ou 3 larves; dans les moyennes, on en trouve souvent jusqu'à une dizaine. Les grosses en contiennent plus d'une vingtaine. Celle qui est représentée (fig. 1, pl. XI) contenait 26 larves.

Toutes ces tumeurs prennent des aspects différents suivant l'àge de leur formation et le stade d'évolution des larves parasites. Celles où les larves sont encore jeunes sont constituées par un tissu fibreux disposé régulièrement autour des loges remplies par les larves. Quand celles-ci commencent à sortir, les loges se transforment en cavités irrégulières remplies par les mues chitineuses et par un pus abondant. Il reste toujours des traces des anciennes loges abandonnées par les larves et comblées par un tissu moins régulier. Leur aspect sur une section est marbré avec des plaques rougeâtres ; ces marbrures sont dues aux anciennes zones hémorragiques et aux petites zones pigmentées en noir par la chitine des mues non résorbées et incluses maintenant dans le tissu néoformé. Nous ne savons pas ce que deviennent par la suite ces tumeurs, mais nous supposons qu'elles peuvent être de nouveau envahies par de jeunes larves qui arrivent dans le duodenum en même temps que des larves âgées en sortent. C'est par un processus pareil que doivent se former les grosses tumeurs.

Nous regrettons de ne pas avoir pu faire une étude histologique plus détaillée de ces tumeurs. La figure que nous donnons d'une coupe (fig. 3, pl. XI) dans la masse d'une de ces tumeurs montre la disposition régulière du tissu fibreux autour d'une larve secondaire qui, ayant une double cuticule, est en train de passer au stade suivant.

Au point de vue de l'action pathogène, nous croyons que ces parasites doivent produire dans le fonctionnement des organes des troubles d'autant plus graves que les tumeurs sont plus volumineuses et qu'elles sont situées plus près du sphincter pylorique.

Caractères qui différencient la larve de Gastrophilus meridionalis de celle de $\boldsymbol{G}$. nasalis. - Comme chez presque tous les animaux examinés cette larve de gastrophile était accompagnée de la larve de $G$. nasalis, nous croyons utile de donner ici quelques détails sur les caractères qui différencient ces deux larves (fig. 3 et 4), 
Tout d'abord, les larves au $3^{\circ}$ stade de $G$. nasalis, bien qu'elles se trouvent fixées à la muqueuse duodénale à côté des autres larves, ne s'enfoncent jamais dans la profondeur des tissus. Sur les animaux provenant de France ou d'Europe centrale et qui présentent des larves de G. nasalis, il n'y a jamais de tumeurs dans la paroi du duodenum. Lorsque les deux sortes de larves sont fixées l'une à côté de l'autre et qu'on essaie de les arracher, celles de G. nasalis se détachent plus facilement parce qu'elles se fixent toujours superficiellement en enfonçant la tête et un segment suivant. Au contraire, les larves de G. meridionalis se laissent difficilement arracher parce

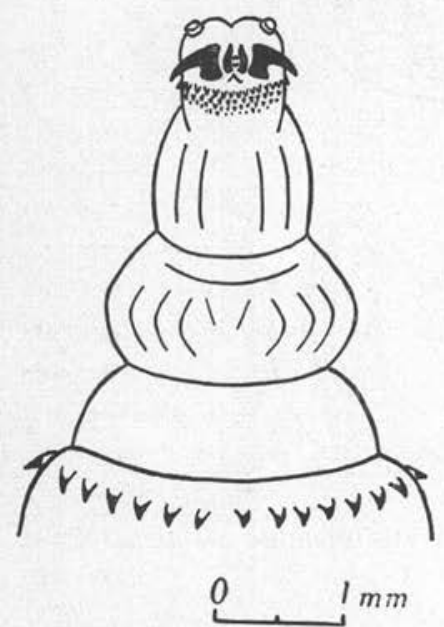

Fıg. 3. - Tête et partie antérieure du corps de la larve de $G$. meridionalis au $3^{\text {e }}$ stade, face ventrale.

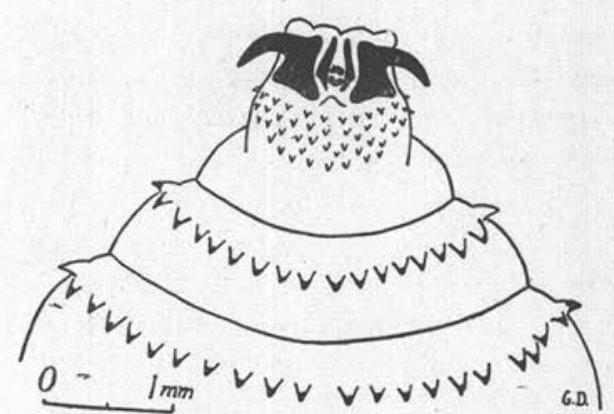

Fig. 4. - Larve de Gastrophilus nasalis, mêmes parties.

qu'elles enfoncent dans la paroi la tête et 3 ou 4 segments suivants.

La couleur des larves vivantes est bien différente d'une espèce à l'autre. Celles de $G$. nasalis ont une couleur jaunâtre et le tégument est opaque ; celles de $G$. meridionalis sont plus claires et, la cuticule étant transparente, la couleur des organes internes et du sang ingéré leur donne un reflet rouge marbré. Chez G. nasalis, tous les segments du $2^{\circ}$ au $11^{\circ}$ sont pouvus d'épines, tandis que chez G. meridionalis le $3^{\circ}$ et le $4^{\circ}$ segment sont complètement nus.

Pour les larves au $2^{\circ}$ stade, les caractères sont encore plus différents. D'abord, les larves de G. meridionalis, sorties des tumeurs, sont d'une couleur plus claire et presque immobiles; celles de G. nasalis, arrachées de la paroi, sont très actives. Les orifices stigmatiques sont normalement développés chez $G$. nasalis, tandis que 
chez G. meridionalis, ils sont très réduits (M, fig. 6). De même, les épines tégumentaires sont presque atrophiées chez cette dernière espèce.

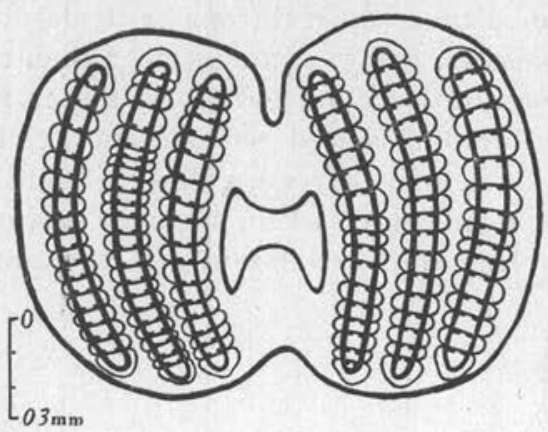

Fif. 5. - Stigmates de la larve de Gastrophilus meridionalis au 3. stade.

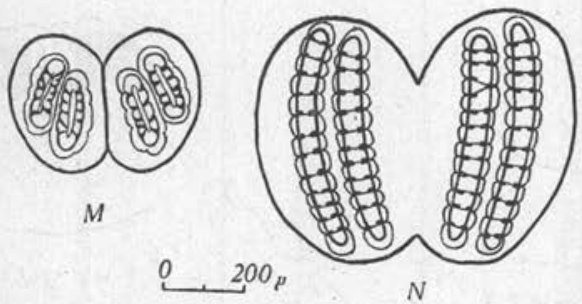

Fig. 6. - Stigmates des larves au $2^{e}$ stade : M, Gastrophilus meridionalis, $\mathrm{N}, G$, nasalis.

\section{Explication des Pi.anches X et XI}

\section{Planche X}

Fig. 1. - Larve de Gastrophilus meridionalis an 3 stade, faces dorsale et ventrale. Fig. 2, - Larve de G. meridionalis au $2^{\circ}$ stade, dans les tumeurs duodénales.

Fig. 3. - Larve de G. meridionalis, mue du $2^{\circ}$ stade et larve au $3^{\circ}$ stade sortie de cette mue. Ces trois figures presque grandeur naturelle.

Fig. 4. - Tumeur de grandeur moyenne sectionnée perpendiculairement à la surface de l'intestin (coupe transversale).

Fig. 5. - Tumeur découverte du côté de la lumière de l'intestin.

Fig. 6. - Tumeur de petite dimension avec 3 larves.

Fig. 7. - Tumeur avec une seule larve.

Fig. 8. - Partie d'une tumeur où on voit de jeunes larves en train de pénétrer à l'intérieur de celle-ci.

\section{PI.ANChe XI}

Fig. 1. - Tnmeur gastrophilienne dans la paroi duodénale, vue à la surface interne de l'intestin.

Fig. 2. - Même tumeur sectionnée perpendiculairement à la paroi intestinale. Ces deux figures sont réduites de $1 / 4$.

Frg. 3. - Coupe histologique dans une portion de tumeur, la larve présente deux téguments (2 et 3 , larve en train de muer). 
ANNALES DE PARASITOLOGIE

T. IX, No 6, Novembre 1931.

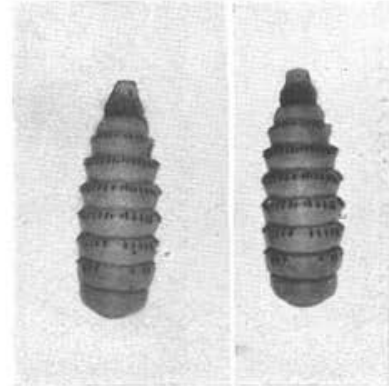

Fig. 1.

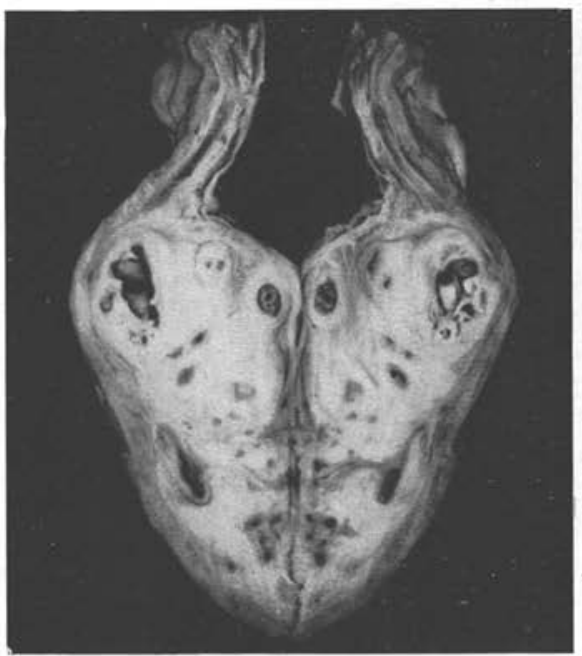

Fig. 4.

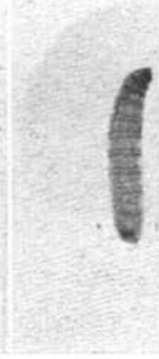

Fig. 2,
Planche $\mathrm{X}$

(Mémoire Dinulescu).

Fit. 3 .

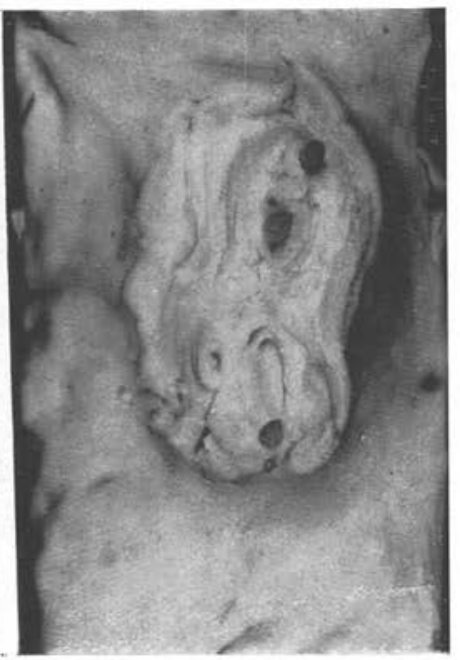

FIG. 5.

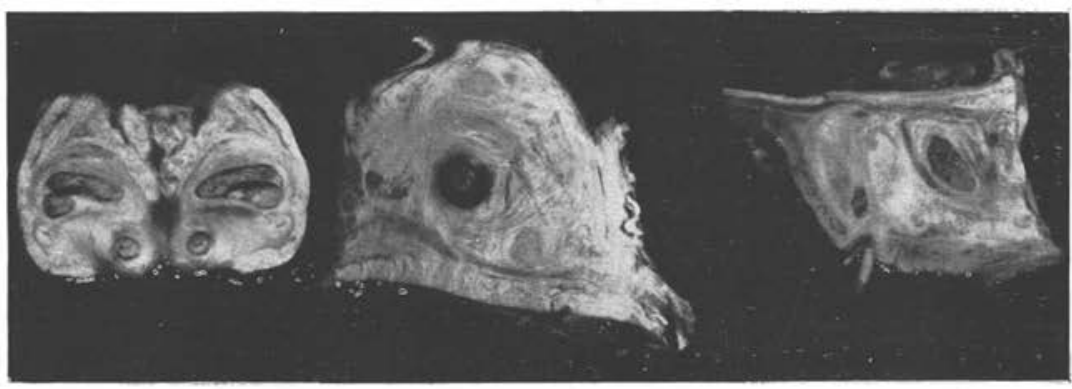

FIG. 6 .

Fig. 7.

Fig. 8.

Masson et Cie, Editeurs 

ANNALES DE PARASITOLOGIE

T. IX, No 6, Novembre 1931.

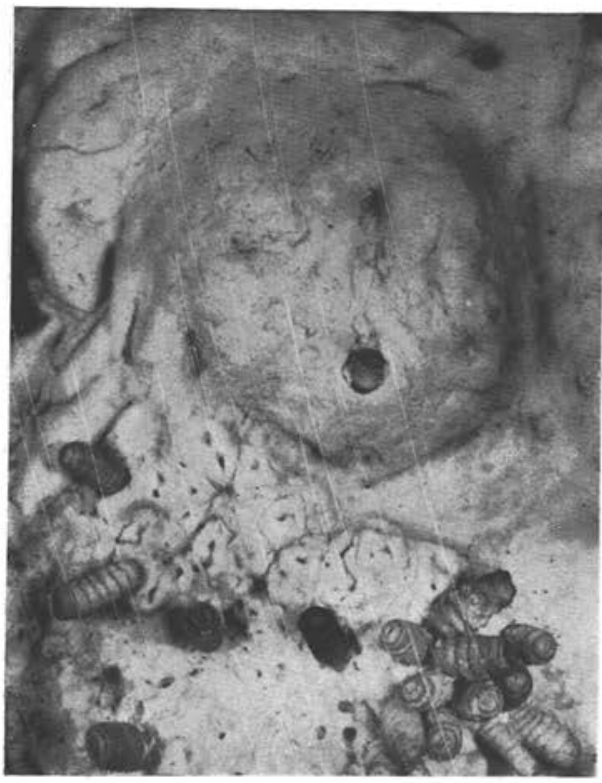

FIG. 1.
Planche XI

(Mémoire Dinulescu).

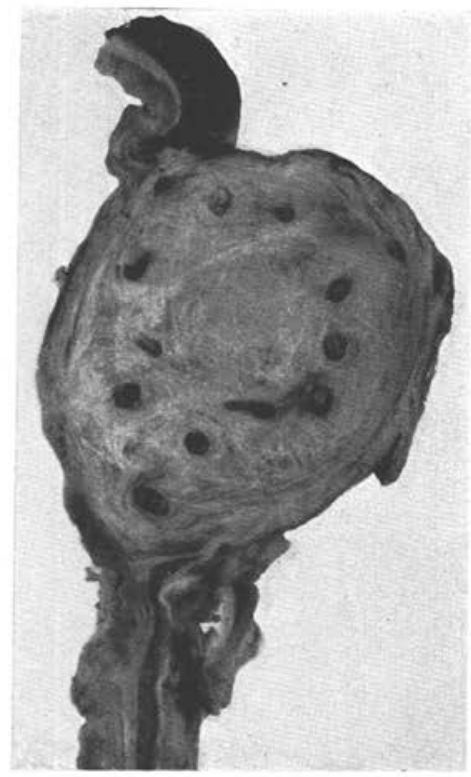

FIG. 2.

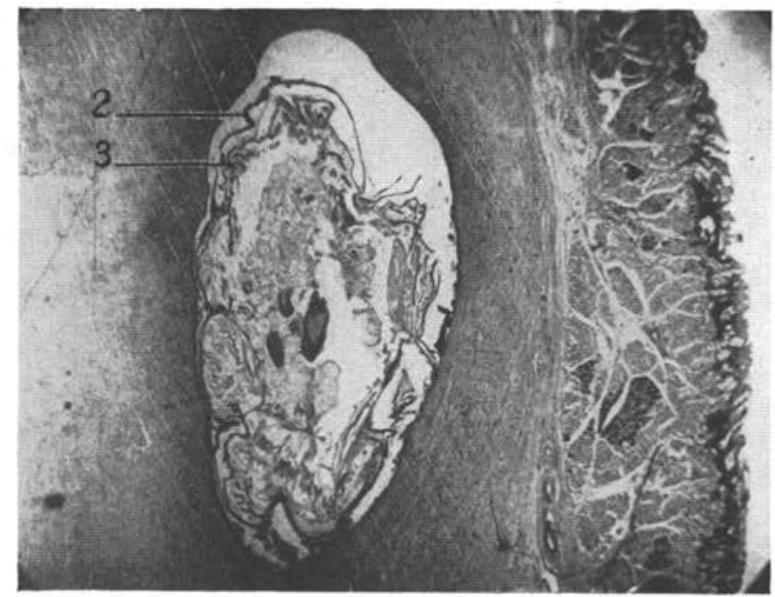

Fig. 3. 



\section{RÉSUMÉ}

Dans ce travail, nous signalons la présence, chez des chevaux d'Espagne, d'une larve de Gastrophilus probablement identique à la forme larvaire d'Wstrus (Gastrophilus) meridionalis Pillers et Ewans, signalée par ces auteurs comme parasitant l'estomac des zèbres en Afrique du Sud. Cette larve, qui se fixe à la paroi dı duodenum, présente, à la différence de toutes les autres larves d'œestrides, la particularité de passer une partie de sa vie (durant le $2^{\circ}$ stade) dans la profondeur des tissus, donnant lieu ainsi à la formation de tumeurs parasitaires dans le duodénum et même le pylore.

Nous donnons des détails sur l'évolution de cette larve pendant deux stades de sa vie. Au $2^{\circ}$ stade, elle s'enfonce dans les tissus de la paroi duodénale en provoquant la formation de tumeurs; elle y mue et sort ensuite au $3^{\circ}$ stade pour continuer, fixée à la surface interne de l'intestin, sa vie jusqu'à la nymphose.

Nous terminons par la description morphologique de la larve à ces deux stades et par une étude sommaire des tumeurs gastrophiliennes.

\section{BiBLIOGRAPHIE}

Brauer (Fr.). - Entomologische Beiträge. Verh. Zocl. Bot. Gesellsch. in Wien, $\mathrm{XIV}, 1864$, p. $891-902$.

Drnulescu (G.). - La larve au 1er stade du G. inermis Br. et la myiase gastrophilienue des joues chez le cheval. Ann. de Parasil., VII, 1929, p. 419-429.

- Sur la biologie des œstres du cheval. C. R. Acad. sc. CXCI, 1930, p. 499-491.

- Une larve d'œstride produisant des tumeurs dans le duodenum des chevaux en Espague. C. R. Acad. Sc., CXCIII, 1931, p. 550-552.

Gurand. - Larve d'œstride trouvée dans une tumeur chez Didelphis murina en Guyane. Bull. Soc. linnéenne du nord de la France, VI, 1874, p. 3.

Goyanez (J.). - Influencia de los parasitos animales en la genesio de cancer, Confer. J. Resen. R. Soc, esp. hist. nat., I, 19_6, p. 197-223.

Hoвueyer (A. et H.). - Morphologie und Biologie der Larve von Gastrophilus pecorum Fbr. Centralbl. f. Bakl., Orig., CVIII, 1928, p. 163-172.

Kemsitz (G. A. V.). - Untersuchungen über den Stoffbestand und Stoffwechsel der Larven von Gastrophilus equi (de Geer.). Zeilschrift f. Biol., physiol . Teil., LXVH, 1917, p. 129.

Pillers (A. W.) et Ewans (A. M.). - A new larva of Gistrus (Gastrophilus) from zebras, Estrus (Gastrophilus) meridionalis. Ann. Trop. Med. and Parasit., $\mathrm{XX}, 1926$, p. 263.

Roubaud (E.). - Autoinoculation et développement primaire dans les muqueuses buccales de la larve du gastrophile équin. C. R. Acad. Sc., CLXIV, 1917. p. 453.

Laboraloire d'évolution des êtres organisés (Prof. Caullery)

Faculté des sciences de Paris. 